\title{
Genotype-specific responses to light stress in eelgrass Zostera marina, a marine foundation plant
}

\author{
Tiina Salo ${ }^{1,2, *}$, Thorsten B. H. Reusch ${ }^{3}$, Christoffer Boström ${ }^{2}$ \\ ${ }^{1}$ Department of Environmental, Social and Spatial Change, Roskilde University, Universitetsvej 1, 4000 Roskilde, Denmark \\ ${ }^{2}$ Department of Biosciences, Environmental and Marine Biology, Åbo Akademi University, Artillerigatan 6, 20520 Åbo, \\ Finland \\ ${ }^{3}$ Evolutionary Ecology of Marine Fishes, GEOMAR, Helmholtz Centre for Ocean Research Kiel, \\ Düsternbrooker Weg 20, 24105 Kiel, Germany
}

\begin{abstract}
Within mono-specific meadows of clonal plants, genotypic diversity may functionally replace species diversity. Little is known about the variability in performance and plasticity of different genotypes towards anthropogenically induced stressors. In this field experiment we compared light-limitation stress responses and recovery of different eelgrass Zostera marina genotypes to assess the variability in phenotypic plasticity and gene expression between different genotypes. Replicated monoculture plots of 4 genotypes were subjected to a simulated turbidity period of $4 \mathrm{wk}$ using shading screens, and their performance during light limitation and $4 \mathrm{wk}$ of recovery was compared to non-shaded controls. In addition to growth and biomass, we investigated storage carbohydrates and quantified the expression of genes involved in carbohydrate metabolism, photosynthesis and control of oxidative stress. Plants showed remarkable plasticity in their stress responses and all phenotypic variables recovered to the control level within $4 \mathrm{wk}$. Depletion and subsequent restoration of sucrose levels differed among genotypes. In terms of gene expression, no consistent patterns were observed. Our study confirms that stress responses and recovery processes can vary substantially between genotypes and the results emphasize the importance of preserving regional genotypic diversity for immediate positive diversity effects and for adaptive evolution in response to global change.
\end{abstract}

KEY WORDS: Gene expression $\cdot$ Plasticity $\cdot$ Seagrass $\cdot$ Shading $\cdot$ Sucrose

\section{INTRODUCTION}

In species-poor communities, genetic diversity may functionally replace species diversity (Hughes et al. 2008). Many aquatic macrophyte stands, such as macroalgal beds, seagrass meadows and saltmarshes, are naturally species-poor while providing the foundation for entire ecosystems. In seagrass meadows, genotypic diversity has been observed to enhance resistance to disturbance (Hughes \& Stachowicz 2004, Ehlers et al. 2008), recovery after a disturbance (Reusch et al. 2005, Hughes \& Stachowicz 2009) and resilience (Hughes \& Stachowicz 2011).
For genotypic diversity to have any ecological effects analogous to species diversity, the genotypes must differ in selectively relevant traits (Hughes et al. 2008). The observed positive effects of genotypic diversity in many studies (see above) implicate differences between genotypes. As genotypes can differ in their morphology and physiology (Hughes et al. 2009, Tomas et al. 2011), it is justified to expect relatively large differences in performance and plasticity between genotypes both during and after stress. Here, we define plasticity as the alteration of the phenotype in the face of environmental change, and any adaptive response aiming to maintain organis- 
mal function is considered plasticity. This may entail 'classical' plasticity, i.e. changes in phenotype within the same genotype, or the maintenance of organismal function despite external stress, also termed buffering (Reusch 2014 and references therein).

Global change and other anthropogenic impacts are altering environmental settings around the world. Whether different plant populations can survive in, and adapt to, these changed conditions depends on the plasticity and adaptive capacity of the genotypes. Due to genetic variability, individuals and genotypes within the same population may differ in their responses to environmental changes (Jump \& Peñuelas 2005). As plasticity in gene expression, i.e. variation in expression of a gene in response to stress, is known to be at least partly heritable and can be affected by natural selection (Whitehead \& Crawford 2006), the variation among genotypes is a prerequisite both for species persistence and for adaptive evolution to occur.

Our model species eelgrass (Zostera marina L.) is a marine foundation plant that serves as a basis for productive and resilient coastal ecosystems throughout the northern hemisphere (den Hartog 1970, Boström et al. 2014). Eelgrass can reproduce either sexually or asexually; some of the range edge populations have been shown to rely almost exclusively on clonal growth, indicated by extremely low clonal richness (Olsen et al. 2004, Reusch \& Boström 2010). Long-term survival of edge populations with low genetic diversity (Reusch et al. 1999, Reusch \& Boström 2010) indicates large plasticity and/or differences between eelgrass genotypes.

In this study, we investigated how individual eelgrass genotypes differed in their tolerance and recovery to light stress imposed by eutrophication. Increased plankton blooms (HELCOM 2009) in combination with increasing amounts of drifting algal mats (Bonsdorff et al. 1997, Norkko et al. 2000) alter the light regime and can cause extensive shading with up to $90 \%$ reduction in light levels (Rasmussen et al. 2012). Shading by drifting algae mats has thus been suggested to be one of the factors responsible for declines in seagrass coverage in subtidal eelgrass populations (Baden et al. 2003, Rasmussen et al. 2012).

In addition to the unresolved issues of possible differences between genotypes, the coupling between genotypes and phenotypes is largely unexplored especially in marine ecosystems (Sultan 2004, Reusch 2014, but see Whitham et al. 2006 for examples in terrestrial communities). We studied eelgrass responses related to carbohydrate metabolism and production at both the physiological and gene expression level, and make the first attempt to relate gene expression and phenotypic responses-in terms of carbohydrate metabolism and productivity - in seagrasses. Understanding regulation of soluble carbohydrates is crucial, as they function as energy reserves in leaves, roots and rhizomes and can be metabolized when extra energy is needed. For example, seasonal changes (Touchette \& Burkholder 2007), low light levels (Gustafsson \& Boström 2013, Villazán et al. 2013), high nitrate (Touchette \& Burkholder 2007) and ammonium concentration (Tomas et al. 2011, Villazán et al. 2013) can lead to increased mobilization of sucrose reserves and, thus, reduced sucrose levels in seagrasses. While there is indication that some of the key enzymes participating in sucrose metabolism are affected by environmental factors (Touchette \& Burkholder 2000, 2007), the exact mechanisms regulating soluble carbohydrate metabolism in seagrasses experiencing environmental stress are not yet known. We hypothesized that the different eelgrass clones differ in their responses to shading stress and in their recovery processes on many different levels from gene expression to phenotypic performance. We also expected storage carbohydrates and plant growth to show similar patterns as the expression of genes related to carbohydrate metabolism and photosynthesis. For more detailed hypotheses for expression of the studied genes, see Table 1.

\section{MATERIALS AND METHODS}

\section{Study area and genotypes}

Eelgrass plants used in this study were collected at Sandön $\left(60^{\circ} 02.164^{\prime} \mathrm{N}, 22^{\circ} 20.47^{\prime} \mathrm{E}\right.$, SW coast of Finland, northern Baltic Sea). Earlier work has indicated that Sandön is one of the few known areas in the Archipelago Sea with a relatively high diversity of eelgrass genotypes (T. B. H. Reusch \& C. Boström unpubl. data), thus this site was chosen as a donor area. To identify possible donor clones and to ensure that these clones represented different genotypes, DNA samples from 13 eelgrass patches (diameter $>2.5 \mathrm{~m}$ ) were collected at 1.9 to $3.0 \mathrm{~m}$ depth. Each sampled patch was marked with ID tags to enable relocation of the chosen genotypes. Between 10 and 20 plant samples were randomly collected both from the center and the edge of each patch. Genotyping to identify unique clones was conducted using 9 polymorphic microsatellite loci (Reusch \& Boström 2010). 
Four monoculture patches of a single genotype each (i.e. a clone) were finally chosen as donor clones. To minimize differences in light acclimatization and to ensure a sufficient amount of plant material for the experiment, these genotypes were chosen based on their similar morphology, depth distribution (ca. $2 \mathrm{~m}$ depth) and adequate patch size (diameter $>3 \mathrm{~m}$ ).

\section{Experimental design}

In June 2011, eelgrass ramets (i.e. modules composed of roots, a piece of rhizome and one leaf bundle) were carefully collected from each of the 4 clones and transported to the experimental site at Fårö $\left(59^{\circ} 55.219^{\prime} \mathrm{N}, 21^{\circ} 47.711^{\prime} \mathrm{E}\right)$. A detailed site description can be found in Salo et al. (2009). Plants were kept submerged during all processing and transport. The experiment was conducted in a homogenous, bare sand area shoreward of a natural eelgrass meadow. Prior to the experiment, single shoots of natural vegetation (both above- and belowground parts) were removed from the experimental area. Plants from different clones were transplanted in replicated $(n=10)$ monoculture plots at 1.7 to $2.2 \mathrm{~m}$ depth in 2 rows parallel to the shore. In total, 40 plots were established, each consisting of 25 ramets. The distance between any 2 neighboring plots was at least $2 \mathrm{~m}$ and the internal position of each genotype and treatment was randomized.

Irradiance at canopy height and water temperature (Fig. 1) were measured every $2 \mathrm{~h}$ using light and temperature loggers (HOBO Pendant ${ }^{\circledR}$, 8K, Onset). The loggers were calibrated for midday hours (10:00 to $14: 00 \mathrm{~h})$ by calculating linear regression of LN light levels in photosynthetic photon flux density (PPFD) during mid-day hours (measured with $4 \pi$ spherical underwater quantum sensor, Li-1935A, Li-Cor) and LN light levels in LUX (HOBO loggers).

The experimental plant plots were allowed to acclimatize and establish themselves for $4 \mathrm{wk}$. As the plants looked healthy and the growth rates were equivalent to growth rates of eelgrass shoots in the adjacent meadow, this period was considered adequate for the plants to recover from transplantation stress. After $4 \mathrm{wk}$, half of the replicate plots for each genotype ( $\mathrm{n}=5$ for each genotype) were exposed to shading that lasted for $4 \mathrm{wk}$. Shading was achieved by anchoring $1 \mathrm{~m}^{2}$ PVC frames covered with thin tarpaulin ca. $50 \mathrm{~cm}$ above the sediment surface, decreasing light intensity by $78 \%$ compared to the ambient conditions. Because stress effects may first become visible weeks after the disturbance (Franssen et al. 2011), we also studied delayed effects during a $4 \mathrm{wk}$ recovery period (Fig. 1). To assess the effects of shading and recovery, the rest of the replicate plots ( $\mathrm{n}=5$ for each genotype) were left to grow in the ambient light conditions and were considered as control plots. Thus, this was a fully crossed repeated measures experimental design, with 4 clones $(1,2,3,4)$ fully crossed with 2 light treatments (shaded and control) and with 2 time points (shading and recovery). At the end of shading and recovery periods, a subsample (3 ramets) from each replicate plot was collected for determination of biomass, growth, plant tissue sucrose content and relative gene expression.

\section{Phenotypic response variables}

To measure leaf production, plants were marked $7 \mathrm{~d}$ prior to sampling using the plastochrone method

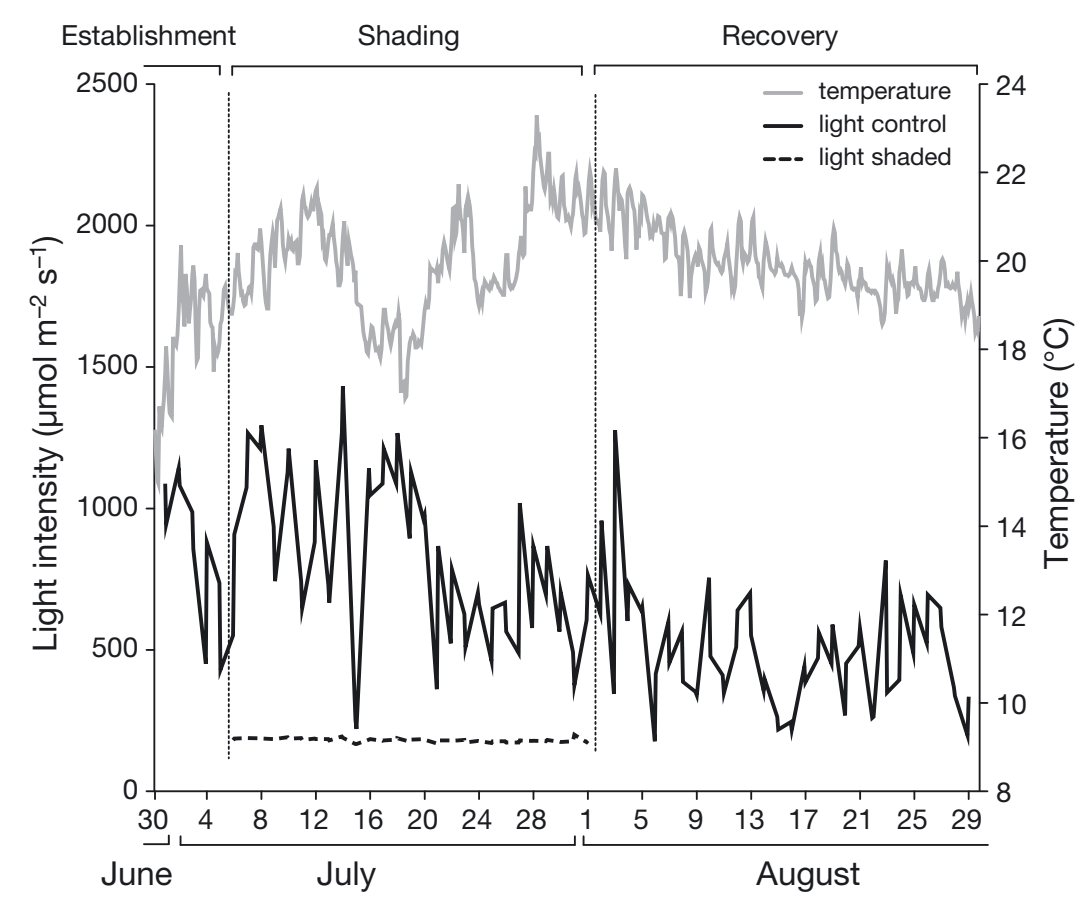

Fig. 1. Temperature and light intensity during shading and recovery in experimental plots. The grey line indicates water temperature (right $y$-axis) and the solid and dashed black lines indicate average mid-day irradiance levels (left $y$-axis) at the control and shaded experimental plots, respectively 
by punching a hole through the leaves within the leaf sheath (Sand-Jensen 1975). Growth was measured as mg (dry weight) new plant material shoot ${ }^{-1} \mathrm{~d}^{-1}$. Sucrose concentrations in freeze-dried and ground plant material were analyzed separately for shoot, root and rhizome tissues. The samples were boiled in $90 \%$ ethanol, after which the extracts were evaporated, re-dissolved and analyzed spectrophotometrically using the resorcinol-hydrocloric acid method (Huber \& Israel 1982). For gene expression analysis, a piece of the youngest or second youngest leaf was submersed in RNAlater (Qiagen) immediately after sampling. Samples were kept at room temperature for $24 \mathrm{~h}$, where after stored at $-20^{\circ} \mathrm{C}$ until analyzed. The number of replicates was 5 for growth and shoot biomass and 3 for sucrose.

The effects of shading, genotype and time on growth, shoot biomass and sucrose concentrations were assessed using a permutational repeated measures approach (Anderson et al. 2008) in PERMANOVA+ (v. 1.0.3) package in PRIMER 6 (v. 6.1.13). Treatment (Shaded, Control), Genotype (1, 2, 3, 4) and Time (Shading, Recovery) were considered as fixed factors, and each plot was nested as a random factor under Treatment and Genotype. Growth, aboveground biomass and sucrose concentrations in shoot, root and rhizome tissues were analyzed in separate univariate analyses. Analyses with significant (at $\alpha=0.05$ ) results were followed by permutational pairwise testing. Resemblance matrixes were based on Euclidean distance and multidimentional scaling (MDS) plots were used to visually inspect the dispersion of multivariate data. All analyses were conducted with 9999 permutations.

The phenotypic plasticity of each clone in terms of sucrose depletion and production in shoot tissue and relative growth rates during shading and recovery was inspected using a reaction norm approach, i.e. a regression between data points from both control and shaded plots was calculated for each genotype separately (Sultan 2003, Nicotra et al. 2010). The regression slopes indicate whether responses are due to differences in genotypes, environmental settings or both. Differing intercepts suggest differences among genotypes, while slope indicates environmental effect on plant performance. If both intercept and slope differ, then genotype and environment have an interactive effect on plant performance. The slope is also a measure of plasticity, where the most positive slope indicates greatest plasticity and least positive slope indicated lower plasticity (Nicotra et al. 2010), while a neutral slope is a sign of phenotypic buffering (Reusch 2014). Autocorrelation of the data were inspected using the Durbin-Watson test (SPSS v. 20.0), and regression slopes for each parameter were compared by calculating $z$-values from r-values in GraphPad Prism 5 (Zar 1996).

\section{Gene expression}

Ten new target genes were identified and selected for gene expression analysis along with 12 target genes previously identified by Bergmann et al. (2010) and Winters et al. (2011) (Table 1). Because severe shading reduces the rate of photosynthesis and increases the mobilization of sugars and other storage carbohydrates, genes involved in carbohydrate metabolism (both biosynthesis and catabolism) were targeted. Changes in light intensity can also induce changes in the photosynthetic apparatus and alter the oxidative stress level for plants, so genes linked to photosynthesis and proteins that function as molecular chaperones or antioxidants (Table 1) were also targeted. Gene expression was tested in both shaded and control plants during shading and recovery. The eukaryotic initiation factor $4 \mathrm{~A}$ was used as a housekeeping gene (Ransbotyn \& Reusch 2006), i.e. a gene with constant expression level that can be used to normalize target gene expression levels. For information about selection criteria for target genes and primers, see the Supplement at www.int-res.com/ articles/suppl/m519p129_supp.pdf.

The expression of target genes was analyzed with BioMark HD System (Fluidigm) using 96.96 Dynamic Array IFC-chip. Four technical replicates were analyzed for each sample. The relative gene expression levels were calculated as:

$$
\begin{gathered}
-\Delta \mathrm{CT}=\mathrm{CT} \text { (housekeeping gene) }-\mathrm{CT} \text { (target gene) (1) } \\
-\Delta \Delta \mathrm{CT}=-\Delta \mathrm{CT} \text { (treatment) }-[-\Delta \mathrm{CT} \text { (control) }]
\end{gathered}
$$

If the technical variation between the quadruplicates was $>0.15$, then the most differing technical replicate was eliminated.

The heat maps for average gene expression were created with the R package 'Heatplus' (Ploner 2012) and the histograms were based on Euclidean distances. The differences in gene expression among genotypes and between time points were tested by analysis of similarity (ANOSIM). The sub-package 'Similarity percentages (SIMPER)' was used to assess the genes contributing most to the dissimilarities between time points. The dispersion of the data was inspected by MDS plots (PRIMER 6). The resemblance matrixes were calculated using Euclidean distances. 
Table 1. Zostera marina genes and primer pairs used in the analysis and their function. H: indicates our hypothesis for the relative expression of the gene in question during shading and recovery, respectively

\begin{tabular}{|c|c|c|c|c|c|}
\hline Gene name & Code & Function & shading & H: & Primer sequences \\
\hline $\begin{array}{l}\text { Eukaryotic initiation } \\
\text { factor } 4 \mathrm{~A}^{\mathrm{a}}\end{array}$ & eIF4A & $\begin{array}{l}\text { Translation } \\
\text { initiation factor }\end{array}$ & - & - & $\begin{array}{l}\text { F: } 5 \text {-TCT TTC TGC GAT GCG AAC AG-3 } \\
\text { R: 5-TGG ATG TAT CGG CAG AAA CG-3 }\end{array}$ \\
\hline Catalase $^{\mathrm{a}}$ & CAT & Antioxidant & down-reg. & up-reg. & $\begin{array}{l}\text { F: 5-ACA AAA TTC CGT CCG TCA-3 } \\
\text { R: 5-GTC CTC AAG GAG TAT TGG TCC TC-3 }\end{array}$ \\
\hline Ascorbate peroxidase $^{a}$ & APX & Antioxidant & down-reg. & up-reg. & $\begin{array}{l}\text { F: 5-GGT GAT TTC TAC CAG CTT GC-3 } \\
\text { R: 5-GAT CCG CAC CTT GGG TA-3 }\end{array}$ \\
\hline $\begin{array}{l}70 \mathrm{kDa} \text { peptidyl-propyl } \\
\text { isomerase }^{\mathrm{b}}\end{array}$ & PPIM & Catalytic activity & down-reg. & up-reg. & $\begin{array}{l}\text { F:5-TAT GCA CCA AGG TGC TGG A-3 } \\
\text { R:5-TTC CAC TTT AAC ATC CCT GTT G-3 }\end{array}$ \\
\hline $\begin{array}{l}\text { Luminal binding } \\
\text { protein }^{\mathrm{b}}\end{array}$ & BIP & $\begin{array}{l}\text { Luminal binding } \\
\text { protein }\end{array}$ & down-reg. & up-reg. & $\begin{array}{l}\text { F: 5-AAT GGT TCT TGC TGG ATG TTT-3 } \\
\text { R: 5-CCC ATG ATG GTG TGA TAC GA-3 }\end{array}$ \\
\hline $\begin{array}{l}\text { Superoxidase } \\
\text { dismutase }(\mathrm{Mn})^{\mathrm{a}}\end{array}$ & SOD & Antioxidant & down-reg. & up-reg. & $\begin{array}{l}\text { F: 5-ATG GGT GTG GCT TGC TTA-3 } \\
\text { R: 5-ATG CAT GCT CCC ATA CAT CT-3 }\end{array}$ \\
\hline $10 \mathrm{kDa}$ chaperonin ${ }^{\mathrm{b}}$ & $10 \mathrm{kDa}$ & $\begin{array}{l}\text { Molecular } \\
\text { chaperone }\end{array}$ & down-reg. & up-reg. & $\begin{array}{l}\text { F: 5-CGT CAC TCC CAA GTA TAC TAC TGT C-3 } \\
\text { R: 5-CTC CGG TCG GAA CAC TGA-3 }\end{array}$ \\
\hline $\begin{array}{l}\text { Heat shock protein } 60 \text {, } \\
\text { isoform } \mathrm{b}^{\mathrm{b}}\end{array}$ & HSP60b & $\begin{array}{l}\text { Molecular } \\
\text { chaperone }\end{array}$ & down-reg. & up-reg. & $\begin{array}{l}\text { F:5-TGG TTG ATG CTG CAA GTG TT-3 } \\
\text { R:5-CAT TCC TCC TCC AGG CAT T-3 }\end{array}$ \\
\hline $\begin{array}{l}\text { Heat shock protein } 60 \text {, } \\
\text { isoform } \mathrm{a}^{\mathrm{b}}\end{array}$ & HSP60a & $\begin{array}{l}\text { Molecular } \\
\text { chaperone }\end{array}$ & down-reg. & up-reg. & $\begin{array}{l}\text { F: 5-GGT TGA TTC TGC AAG CGT CT-3 } \\
\text { R:5-ATC CCA CCC ATT CCT CCA-3 }\end{array}$ \\
\hline Heat shock protein $70^{\mathrm{b}}$ & HSP70 & $\begin{array}{l}\text { Molecular } \\
\text { chaperone }\end{array}$ & down-reg. & up-reg. & $\begin{array}{l}\text { F: 5-CAC GAC CGT GTT GAG ATC AT-3 } \\
\text { R: 5-ACC GCT TCG CAT CAA AGA C-3 }\end{array}$ \\
\hline Heat shock protein $80^{\mathrm{b}}$ & HSP80 & $\begin{array}{l}\text { Molecular } \\
\text { chaperone }\end{array}$ & down-reg. & up-reg. & $\begin{array}{l}\text { F: 5-GCA ATT CTT CCG ATG CTC TT-3 } \\
\text { R: 5-GCG ATC GTA CCC AAG TTG TT-3 }\end{array}$ \\
\hline Starch synthase & STS & $\begin{array}{l}\text { Enzyme (starch } \\
\text { biosynthesis) }\end{array}$ & down-reg. & up-reg. & $\begin{array}{l}\text { F: 5-GTT TAT AAT GAT TGG CAC ACG G-3 } \\
\text { R: 5-TTA TGG ATG CAG AAT ACA GCC T-3 }\end{array}$ \\
\hline Carbohydrate kinase & C-kin & $\begin{array}{l}\text { Enzyme (carbohy- } \\
\text { drate metabolism) }\end{array}$ & up-reg. & down-reg. & $\begin{array}{l}\text { F: 5-TTT GTT CCC ACT GTC ACT GG-3 } \\
\text { R: 5-CTG ACG AAC CAC CTA GTC TG-3 }\end{array}$ \\
\hline $\begin{array}{l}\text { Fructose-bisphosphate } \\
\text { aldolase }\end{array}$ & $\mathrm{FBiA}$ & $\begin{array}{l}\text { Enzyme (fructose } \\
\text { catabolism) }\end{array}$ & up-reg. & down-reg. & $\begin{array}{l}\text { F: 5-AAG AAT CCA AGG CAA TAC GTG-3 } \\
\text { R: 5-TCC TAA CCA GAG TTA AGG CCA-3 }\end{array}$ \\
\hline Sucrose phospatase & S-phos & $\begin{array}{l}\text { Enzyme (sucrose } \\
\text { biosynthesis) }\end{array}$ & down-reg. & up-reg. & $\begin{array}{l}\text { F: 5-GTT TGA TGT GGG CTT GAC TG-3 } \\
\text { R: 5-AAT CGG GTT GGT ATT TAA ACG G-3 }\end{array}$ \\
\hline Sucrose synthase & SS & $\begin{array}{l}\text { Enzyme (sucrose } \\
\text { catabolism) }\end{array}$ & up-reg. & down-reg. & $\begin{array}{l}\text { F: 5-TTA CCG TAT AAC TCG ACC AAA CC-3 } \\
\text { R: 5-TAG CAA AGA AGA CAA CAC TGA G-3 }\end{array}$ \\
\hline $\begin{array}{l}\text { RuBisCo, large subunit- } \\
\text { binding protein } \\
\text { subunit alpha }\end{array}$ & RuBisCo & $\begin{array}{c}\text { Enzyme (in } \\
\text { photosynthesis) }\end{array}$ & down-reg. & up-reg. & $\begin{array}{l}\text { F: 5-CCA TCT CTA CCG CTA TCC CT-3 } \\
\text { R: 5-GAC GAC CTC ACA ACA AAC CT-3 }\end{array}$ \\
\hline Chlorophyll synthase & Chl_synth & $\begin{array}{l}\text { Enzyme (in } \\
\text { chlorophyll sythase) }\end{array}$ & up-reg. & down-reg. & $\begin{array}{l}\text { F: 5-GTC TCA GAT GCC CTA ACC AC-3 } \\
\text { R: 5-TCC CTC ATA ACT ACT TCA CGA C-3 }\end{array}$ \\
\hline $\begin{array}{l}\text { Photosystem II, } 22 \mathrm{kDa} \\
\text { protein }^{\mathrm{a}}\end{array}$ & $22 \mathrm{kDa}$ & $\begin{array}{l}\text { Photosynthesis, } \\
\text { chloroplast precursor }\end{array}$ & up-reg. & down-reg. & $\begin{array}{l}\text { F: 5-TTC CCA AAA AGG TGG TAG TTA-3 } \\
\text { R: 5-ATA AAG AAG CGG CAA AAC C-3 }\end{array}$ \\
\hline Photosystem II, CP47 & $\mathrm{CP} 47$ & $\begin{array}{l}\text { Photosynthesis, core } \\
\text { antenna complex }\end{array}$ & up-reg. & down-reg. & $\begin{array}{l}\text { F: 5-AGG AAG CAG ATC TAT TCT ATA CCC-3 } \\
\text { R: 5-GTG AGA ATT TCA TTG GAA CGA G-3 }\end{array}$ \\
\hline Photosystem II, CP43 & $\mathrm{CP} 43$ & $\begin{array}{l}\text { Photosynthesis, core } \\
\text { antenna complex }\end{array}$ & up-reg. & down-reg. & $\begin{array}{l}\text { F: 5-CAC CAC ATC TGT TAG AAG AAC C-3 } \\
\text { R: 5-CGC AGC TAT GAC TAT CAT CTC }-3\end{array}$ \\
\hline $\begin{array}{l}\text { Photosystem I, reaction } \\
\text { center subunit IV }\end{array}$ & PSI VI & $\begin{array}{l}\text { Photosynthesis, } \\
\text { chloroplast precursor }\end{array}$ & up-reg. & down-reg. & $\begin{array}{l}\text { F: 5-GGG AAC CAA GGT GAA GAT T-3 } \\
\text { R: 5-GAA TCT CAC CAC AAC TGG GTA-3 }\end{array}$ \\
\hline $\begin{array}{l}\text { Photosystem I, reaction } \\
\text { center subunit VI }\end{array}$ & PSI IV & $\begin{array}{l}\text { Photosynthesis, } \\
\text { chloroplast precursor }\end{array}$ & up-reg. & down-reg. & $\begin{array}{l}\text { F: 5-GTG GTC TAC TGG AAC TAC TAC AC-3 } \\
\text { R: 5-GTG ATC TTG AAG GGA TGT CGG-3 }\end{array}$ \\
\hline
\end{tabular}




\section{RESULTS}

\section{Phenotypic responses}

Shading suppressed eelgrass growth rates by $37 \%$ $(p=0.017$; Table 2, Fig. 2a) and aboveground biomass was ca. $33 \%$ lower in the shaded plots as compared to the control plots ( $p=0.003$; Table 2, Fig. 2c). During recovery, the shaded plants reached the aboveground biomass levels of control plants (pairwise comparisons, $t=4.123, \mathrm{p}=0.0003$ and $t=$ $0.1016, p=0.919$ for shading and recovery, respectively; Fig. 2d).

Shading resulted in an average decrease of $56 \%$ and $69 \%$ in sucrose levels in shoot and root tissue $(p=0.030$ and $p=0.004$, respectively; Table 2, Fig. $3 \mathrm{a}, \mathrm{c})$. Rhizome sucrose levels were affected to a lesser extent $(24 \%)$ and the difference between control and shading treatments was only marginally $(\mathrm{p}=$ 0.054) significant (Table 2, Fig. 3e). Shoot tissue sucrose levels were lower under shading as compared to controls $(t=2.955, \mathrm{p}=0.0085$; Table 2$)$, but recovered to the control level during recovery $(t=$ $0.9148, p=0.380$; Fig. $3 b$ ). Shoot tissue sucrose levels were also affected by both genotype and time (interaction $\mathrm{p}=0.013$, Table 2). In particular, while shoot sucrose levels did not differ significantly between genotypes during shading, Genotype 4 had significantly higher shoot sucrose levels compared to the Genotypes 1,2 and 3 during recovery $(t=6.365, \mathrm{p}=$ $0.0025, t=6.584, \mathrm{p}=0.002$ and $t=2.846, \mathrm{p}=0.037$, respectively; Fig. 3b). In addition, Genotype 2 had higher shoot sucrose levels compared to Genotype 1 $(t=2.518, \mathrm{p}=0.042)$. The genotypes also differed in their rhizome sucrose content when comparing shading and recovery time points $(p=0.0023$; Table 2$)$. During shading, Genotype 2 had significantly higher sucrose levels in rhizome tissue than Genotype $4(t=$ $2.655, p=0.029$; Fig. 3e), while Genotype 1 had significantly lower sucrose levels compared to Genotype 4 during recovery ( $t=3.404, \mathrm{p}=0.02$; Fig. $3 \mathrm{f}$ ). Sucrose levels in root tissues followed the same trend as in shoot tissues (Table 2): sucrose levels declined during shading ( $t=4.641, \mathrm{p}=0.0006$; Fig. $3 \mathrm{c}$ ), while they reached the same sucrose level as in control plants during recovery $(t=3.438, \mathrm{p}=0.974$; Fig. $3 \mathrm{~d})$.

The reaction norm plots (Fig. 4) illustrate that both genotype and light environment affected plant performance. Some of the genotypes exhibited less negative slopes than others (depending on the response variable in question), and hence managed to buffer their performance against stress under low light conditions better than genotypes whose results dis-
Table 2. Univariate repeated measures PERMANOVAs on the impacts of genotype (GE) $(1,2,3,4)$, treatment (TR) (shaded, control), time (TI) (shading, recovery) and plot on leaf growth rates, shoot biomass and sucrose levels in shoot, root and rhizome tissue. Bolded values indicate statistically significant results $(\mathrm{p}<0.05)$

\begin{tabular}{|c|c|c|c|c|}
\hline & $\mathrm{df}$ & MS & Pseudo- $F$ & $\mathrm{p}($ perm $)$ \\
\hline \multicolumn{5}{|l|}{ Growth rate } \\
\hline Genotype & 3 & $3.64 \times 10^{-7}$ & 0.7375 & 0.537 \\
\hline Treatment & 1 & $3.08 \times 10^{-6}$ & 6.2300 & 0.017 \\
\hline Time & 1 & $1.28 \times 10^{-6}$ & 1.9643 & 0.175 \\
\hline $\mathrm{GE} \times \mathrm{TR}$ & 3 & $3.97 \times 10^{-7}$ & 0.8033 & 0.503 \\
\hline $\mathrm{GE} \times \mathrm{TI}$ & 3 & $3.78 \times 10^{-7}$ & 0.5779 & 0.636 \\
\hline $\mathrm{TR} \times \mathrm{TI}$ & 1 & $2.07 \times 10^{-6}$ & 3.1602 & 0.082 \\
\hline Plot $(T R \times G E)$ & 32 & $4.94 \times 10^{-7}$ & 0.7556 & 0.185 \\
\hline $\mathrm{GE} \times \mathrm{TR} \times \mathrm{TI}$ & 3 & $4.07 \times 10^{-7}$ & 0.6221 & 0.604 \\
\hline Residual & 32 & $6.54 \times 10^{-7}$ & & \\
\hline \multicolumn{5}{|l|}{ Shoot biomass } \\
\hline Genotype & 3 & $3.70 \times 10^{-3}$ & 1.1640 & 0.336 \\
\hline Treatment & 1 & $1.59 \times 10^{-2}$ & 4.9973 & 0.031 \\
\hline Time & 1 & $3.07 \times 10^{-3}$ & 2.2896 & 0.142 \\
\hline $\mathrm{GE} \times \mathrm{TR}$ & 3 & $2.67 \times 10^{-3}$ & 0.8398 & 0.480 \\
\hline $\mathrm{GE} \times \mathrm{TI}$ & 3 & $6.75 \times 10^{-4}$ & 0.5034 & 0.690 \\
\hline $\mathrm{TR} \times \mathrm{TI}$ & 1 & $1.40 \times 10^{-2}$ & 10.4670 & 0.003 \\
\hline Plot $(\mathrm{TR} \times \mathrm{GE})$ & 32 & $3.18 \times 10^{-3}$ & 2.3709 & 0.009 \\
\hline $\mathrm{GE} \times \mathrm{TR} \times \mathrm{TI}$ & 3 & $1.59 \times 10^{-3}$ & 1.1870 & 0.332 \\
\hline Residual & 32 & $1.34 \times 10^{-3}$ & & \\
\hline \multicolumn{5}{|l|}{ Shoot sucrose } \\
\hline Genotype & 3 & 7355.60 & 0.6504 & 0.594 \\
\hline Treatment & 1 & 64176.00 & 5.6741 & 0.030 \\
\hline Time & 1 & 84156.00 & 9.2882 & 0.008 \\
\hline $\mathrm{GE} \times \mathrm{TR}$ & 3 & 3510.70 & 0.3104 & 0.813 \\
\hline $\mathrm{GE} \times \mathrm{TI}$ & 3 & 44397.00 & 4.9000 & 0.013 \\
\hline $\mathrm{TR} \times \mathrm{TI}$ & 1 & 97392.00 & 10.7490 & 0.005 \\
\hline Plot $(\mathrm{TR} \times \mathrm{GE})$ & 16 & 11310.00 & 1.2483 & 0.332 \\
\hline $\mathrm{GE} \times \mathrm{TR} \times \mathrm{TI}$ & 3 & 11333.00 & 1.2508 & 0.326 \\
\hline Residual & 16 & 9060.60 & & \\
\hline \multicolumn{5}{|l|}{ Root sucrose } \\
\hline Genotype & 3 & 68.99 & 0.8518 & 0.489 \\
\hline Treatment & 1 & 919.84 & 11.3580 & 0.004 \\
\hline Time & 1 & 212.28 & 2.9201 & 0.105 \\
\hline $\mathrm{GE} \times \mathrm{TR}$ & 3 & 113.15 & 1.3972 & 0.279 \\
\hline $\mathrm{GE} \times \mathrm{TI}$ & 3 & 142.48 & 1.9600 & 0.147 \\
\hline $\mathrm{TR} \times \mathrm{TI}$ & 1 & 944.16 & 12.9880 & 0.002 \\
\hline Plot $(\mathrm{TR} \times \mathrm{GE})$ & 16 & 80.98 & 1.1140 & 0.423 \\
\hline $\mathrm{GE} \times \mathrm{TR} \times \mathrm{TI}$ & 3 & 70.75 & 0.9732 & 0.431 \\
\hline Residual & 16 & 72.70 & & \\
\hline \multicolumn{5}{|l|}{ Rhizome sucrose } \\
\hline Genotype & 3 & 4289.40 & 0.6003 & 0.627 \\
\hline Treatment & 1 & 30455.00 & 4.2624 & 0.054 \\
\hline Time & 1 & 8101.50 & 0.5367 & 0.476 \\
\hline $\mathrm{GE} \times \mathrm{TR}$ & 3 & 9187.20 & 1.2858 & 0.317 \\
\hline $\mathrm{GE} \times \mathrm{TI}$ & 3 & 50411.00 & 3.3394 & 0.047 \\
\hline $\mathrm{TR} \times \mathrm{TI}$ & 1 & 130.21 & 0.0086 & 0.929 \\
\hline Plot $(\mathrm{TR} \times \mathrm{GE})$ & 16 & 7144.90 & 0.4733 & 0.926 \\
\hline $\mathrm{GE} \times \mathrm{TR} \times \mathrm{TI}$ & 3 & 10185.00 & 0.6747 & 0.591 \\
\hline Residual & 16 & 15096.00 & & \\
\hline
\end{tabular}



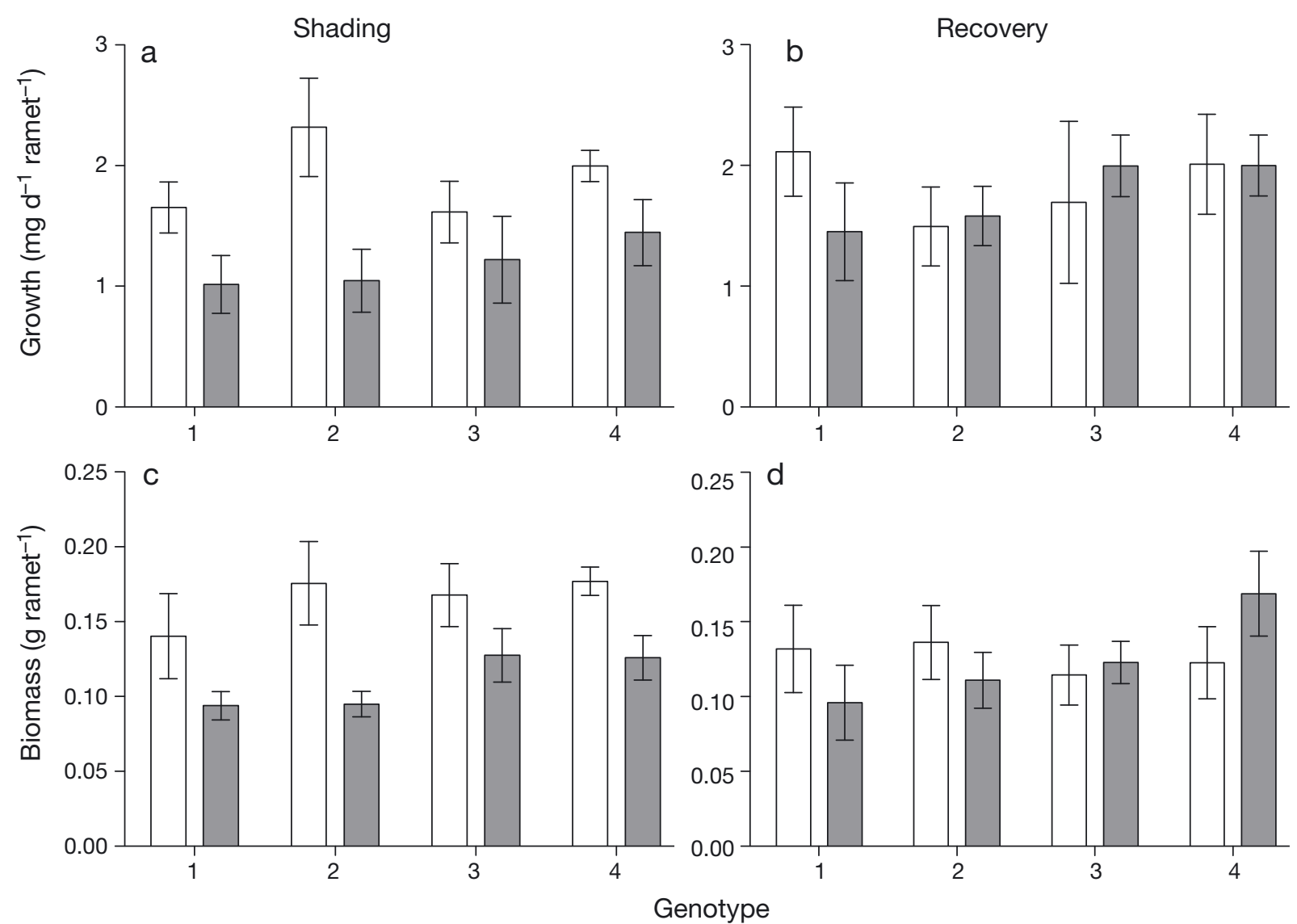

Fig. 2. (a,b) Mean growth and (c,d) aboveground biomass of different genotypes (1-4) after shading (left) and recovery (right) in control (open bars) and shaded treatments (filled bars) $(n=5)$. Error bars are $\pm \mathrm{SE}$

played steeper slopes. Despite the different slopes, the variation within genotypes was too large to yield significant differences in slopes or intercepts (Fig. 4a,c,d). However, shoot sucrose concentrations during recovery indicated that recovery processes differed significantly among genotypes $(p=0.032$; Fig. $4 \mathrm{~b}$ ), and Genotype 2 recovered best in terms of increase in sucrose concentrations.

\section{Gene expression}

The gene expression of the studied genes varied from down-regulated (negative $-\Delta \Delta \mathrm{CT}$ values) to upregulated (positive $-\Delta \Delta \mathrm{CT}$ values), depending on the gene and genotype in question. The relative gene expression profiles differed among the studied genotypes both during shading and recovery (Fig. 5), but due to high intra-genotypic variation these patterns were not significant. The relative gene expression was not significantly different between different genotypes during shading (Global R: 0.021, $\mathrm{p}=0.32$ ) or recovery (Global R: 0.011, p = 0.394). The gene expression differed somewhat more between shading and recovery (Global R: 0.039, $p=0.067$ ) suggesting that the plants altered their gene expression in response to shading. Genes that contributed most to this difference were STS $(11.41 \%)$, SS $(7.94 \%)$, HSP $80(7.89 \%)$, CP43 $(7.23 \%), 22 \mathrm{kDa}(7.04 \%)$, PSI IV (6.48\%) and Sphos $(6.00 \%)$.

Genes linked to carbohydrate catabolism (C-kin, FBiA and SS) were hypothesized to be up-regulated during shading and down-regulated during recovery, while genes linked to carbohydrate synthesis (Sphos and STS) were expected to show the opposite pattern (Table 1). However, the expression of genes linked to carbohydrate catabolism was not consistent among the studied genotypes during either shading or recovery (Fig. 5). The expression of genes related to carbohydrate synthesis also varied between genotypes, but these were expressed more consistently as the shaded plants either up-regulated or downregulated both Sphos and STS simultaneously. Sucrose synthase (SS) and sucrose phosphatase 

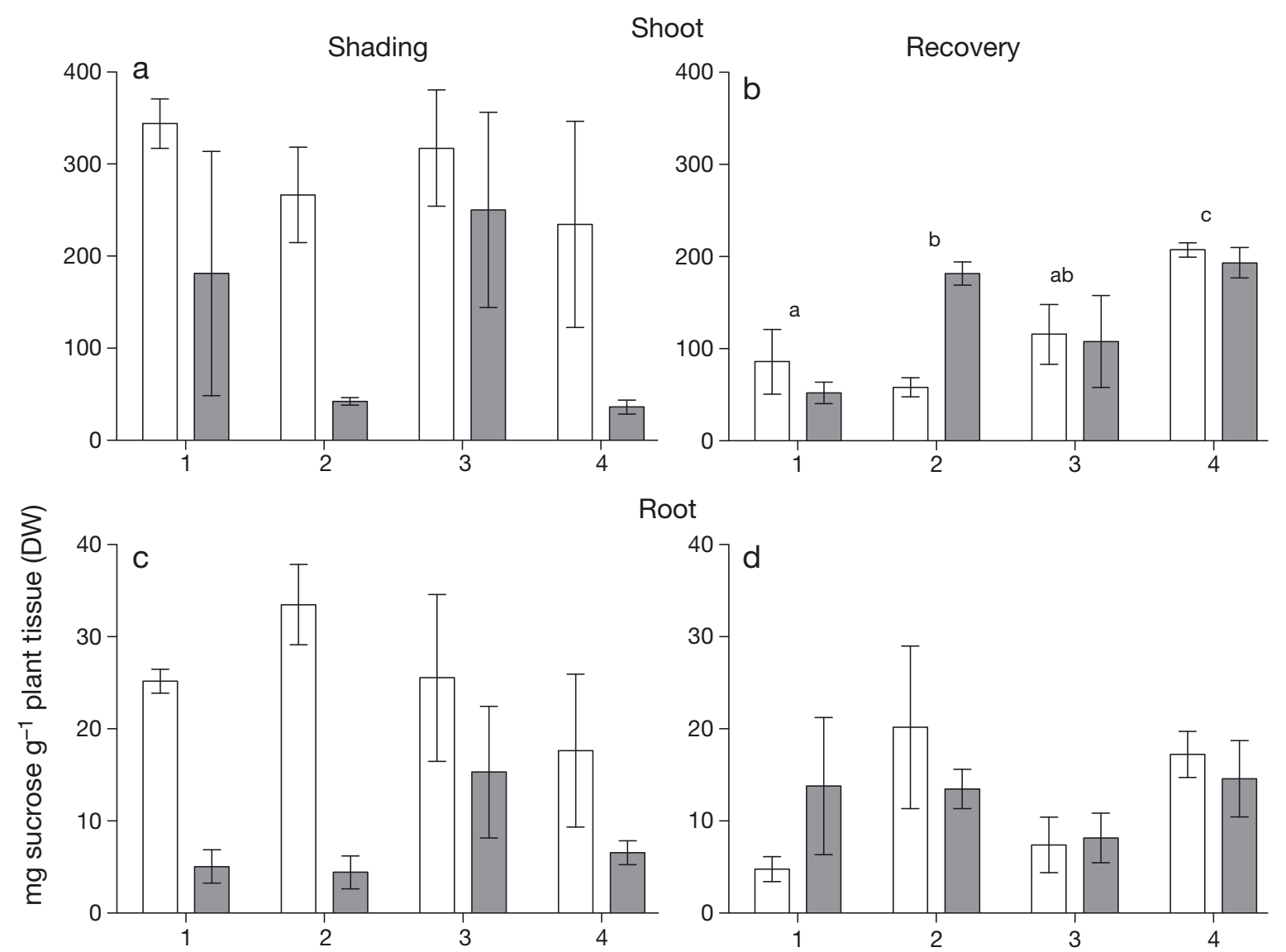

Root

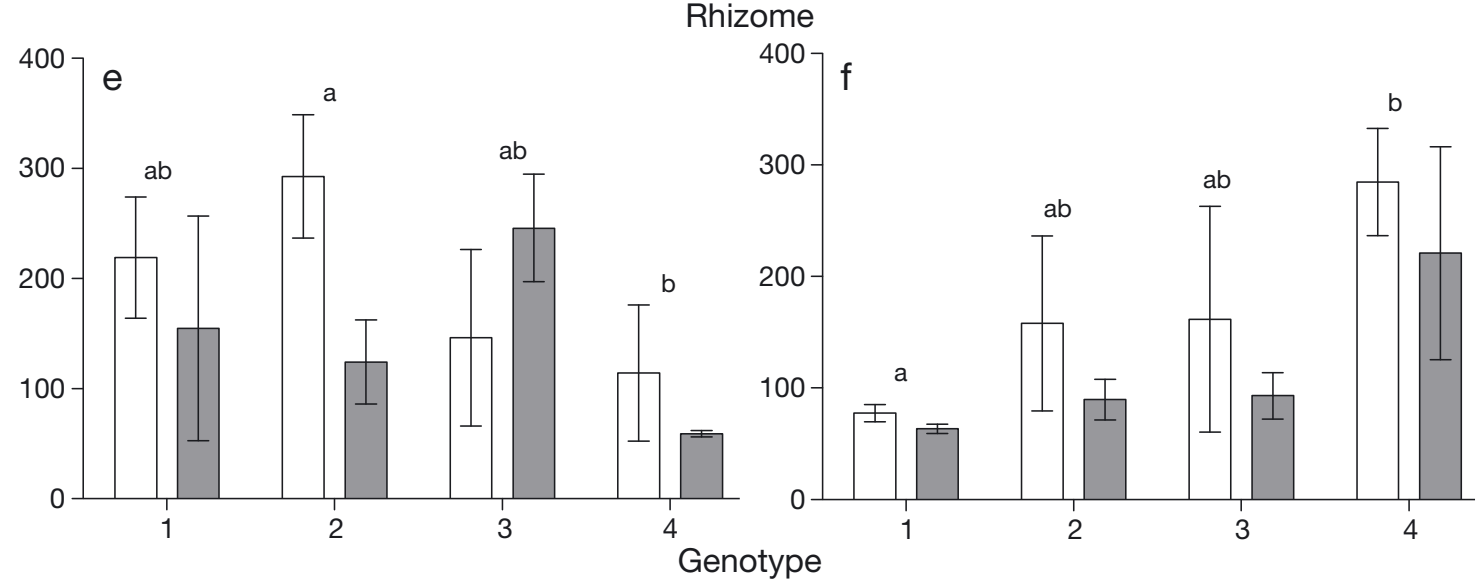

Fig. 3. Mean sucrose concentrations in $(\mathrm{a}, \mathrm{b})$ shoot, $(\mathrm{c}, \mathrm{d})$ root, and $(\mathrm{e}, \mathrm{f})$ rhizome tissue after shading (left) and recovery (right) in control (open bars) and shaded treatments (filled bars) $(n=3)$. The different letters above bars indicate significant differences $(\mathrm{p}<0.05)$ between the genotypes based on permutational pairwise testing. Error bars are \pm SE

(Sphos) are key enzymes in sucrose catabolism and synthesis, respectively. Although non-significant, sucrose concentrations seemed to reflect to up- and down-regulation of SS and Sphos (Figs. 4a,b \& 5).

Most of the genes related to photosynthesis (PSI IV, PSI VI, CP43, CP47, $22 \mathrm{kDa}$, Chl_synth) were expected to be up-regulated during shading and down-regulated during recovery, except for RuBisCo which was expected to show the opposite pattern (Table 1). Interestingly, genotypes responded in contrasting ways to shading and recovery in terms of photosynthesis-related gene expression. Genotype 1 acted as expected by up-regulating most of the photosynthesis-linked genes during shading and 

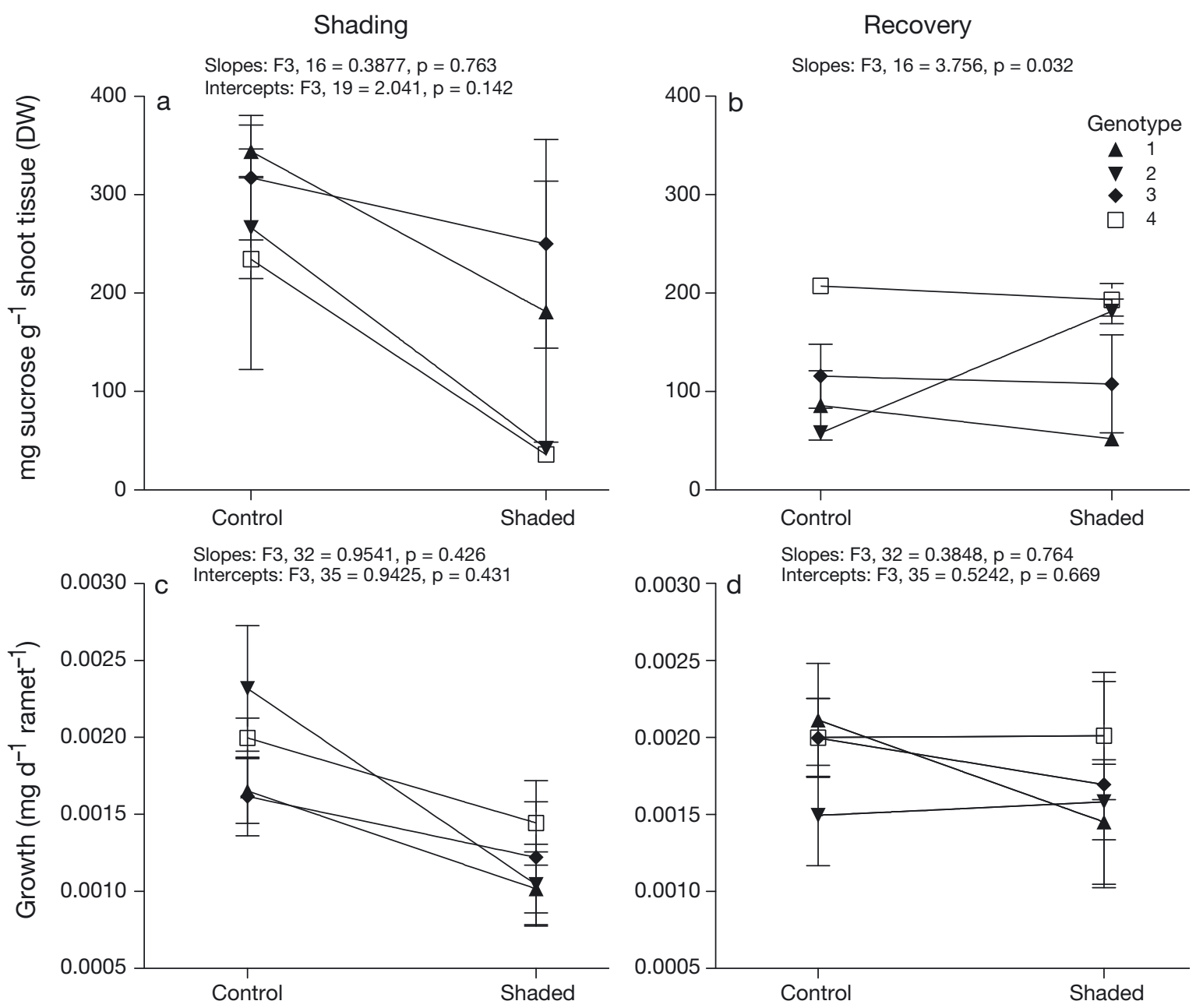

Fig. 4. Reaction norm plots for $(\mathrm{a}, \mathrm{b})$ sucrose levels in shoot tissue and $(\mathrm{c}, \mathrm{d})$ relative growth rate in different genotypes (1-4) during shading (left) and recovery (right) in control (open bars) and shaded treatments (filled bars). Statistical results indicate the differences in slopes and intercepts. A straight line indicates neutral plasticity (i.e. shading had no effect) while positive and negative slopes indicate plastic responses to shading

down-regulating these genes during recovery (Fig. 5), while Genotype 2 showed the opposite pattern. Photosystem II reaction subunit IV and VI (PSI IV and PSI VI) genes are essential for photosynthesis as these units enable light energy to be converted into chemical energy. Although non-significant, reaction norms for growth seem to reflect up- and down-regulation of these genes (cf. Genotypes $1 \& 2$; Figs. 4c,d \& 5).

Due to an expected decrease in oxidative stress in the shaded plots compared to the control plots, genes linked to oxidative stress (CAT, APX, PPIM, BIP, SOD, $10 \mathrm{kDa}, \mathrm{HSP} 60 \mathrm{a}, \mathrm{HSP} 60 \mathrm{~b}, \mathrm{HSP} 70$ and HSP80) were expected to be expressed to a lower degree during shading together with stronger upregulation during recovery (Table 1). However, only Genotypes 1 and 3 down-regulated most of these genes during shading, and no clear up-regulation was obvious during recovery, with an exception of the Genotype 2, which up-regulated all of these genes (Fig. 5).

\section{DISCUSSION}

In clonal organisms, inter-genotypic differences are a fundamental level of biological diversity that is a prerequisite for both adaptive evolution and immediate ecological effects of biodiversity. This study provides evidence that the phenotypes of particular genotypes vary in response to stress. We also found patterns in gene expression (although non-significant) that can be tentatively associated with particular differences in storage carbohydrates and 

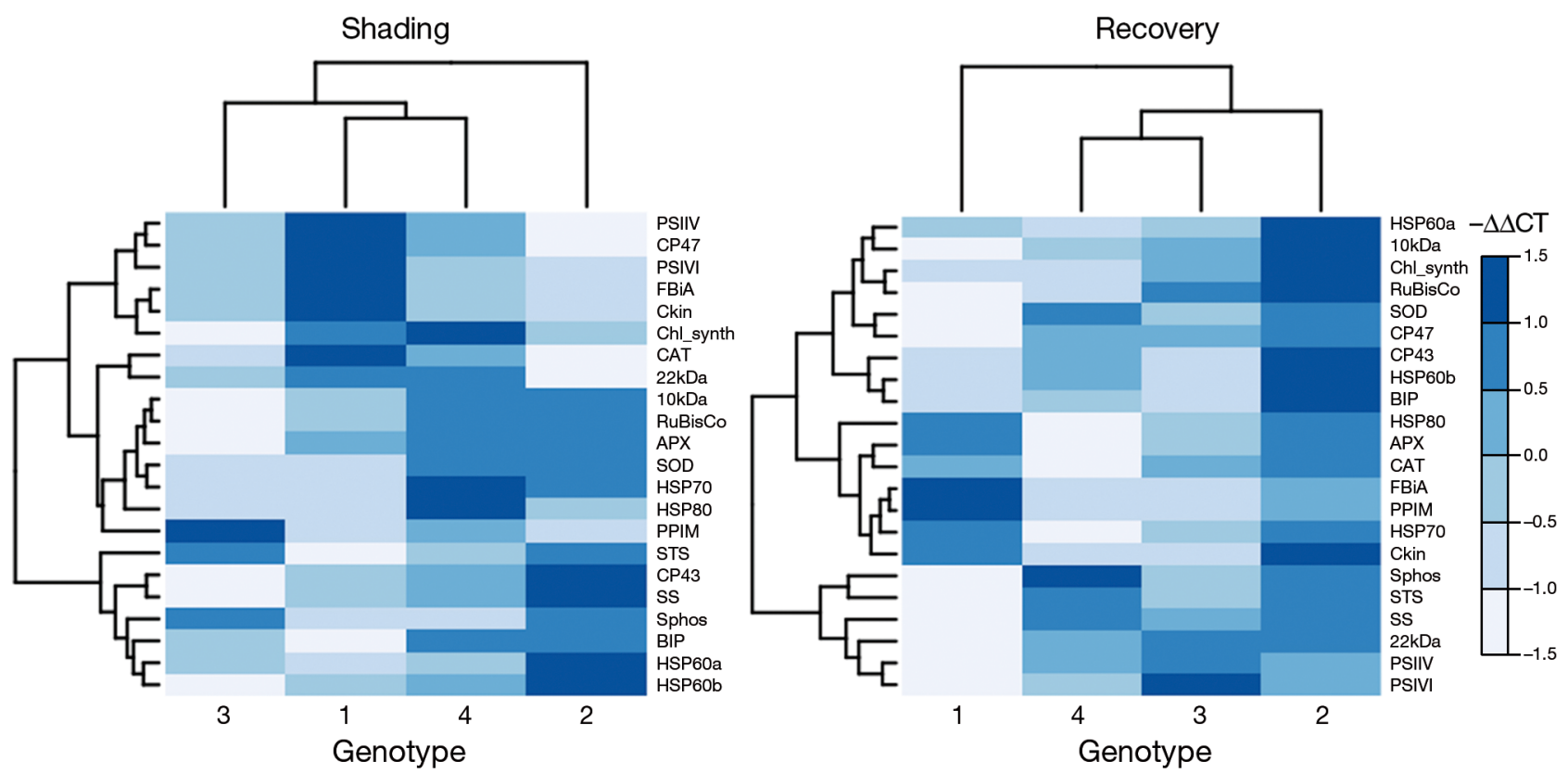

Fig. 5. Heat maps indicating mean relative gene expression $(-\Delta \Delta \mathrm{CT})$ in different genotypes during shading and recovery. Dendrograms indicate the similarity of expression between genotypes ( $x$-axis) and genes ( $y$-axis). Expression is color-coded with white indicating the lowest (down-regulation) and dark blue indicating the highest (up-regulation) gene expression. Gene abbreviations are given in Table 1

growth rates. However, since we were only able to compare 4 genotypes in this study, these results are preliminary.

Fitness-related traits that increase the capacity of organisms to tolerate extreme environmental events are likely to become more important in the future due to global change (Jump \& Peñuelas 2005). Traits of stress tolerance during and after extreme events are still largely under-studied; identifying these key traits and assessing their adaptive plasticity is essential to help predict long-term survival of organisms (Nicotra et al. 2010). In terms of physiological parameters, the experimental plants showed full recovery in their performance at the end of the experiment. As expected during heavy shading, all the genotypes suppressed their leaf growth and carbohydrate levels, while opposite patterns were observed during recovery. The ability to adjust to and survive a long period of extensive shading indicates remarkable plasticity in eelgrass, and such tolerance is highly important for seagrass long-term survival. The few earlier studies assessing variation in seagrass genotypes have found differences in genotypes, for example in biomass production (Hughes et al. 2009, Tomas et al. 2011) and biomass loss due to grazing pressure (Tomas et al. 2011). While these aspects might impact stress tolerance of plants, the latter study found no genotype-specific differences in relation to nitrogen enrichment (Tomas et al. 2011). In this study, some genotypes depleted and restocked their sucrose levels to higher degree than others in response to shading stress and recovery from it, indicating that these genotypes differ, among others aspects, in their carbohydrate metabolism (Figs. $3 \& 4 a, b)$. As carbohydrate reserves and mobilization of these reserves are in a key position for plants to carry on cellular metabolism during stress (Touchette \& Burkholder 2000), the results suggest that some genotypes are likely to be more stress-resistant and recover faster from stress events. In seagrasses this could mean increased resistance in some genotypes to shading caused by planktonic or drifting algae or increased turbidity. However, even high genetic variability in a population does not ensure that the population can keep up with environmental changes (Jump \& Peñuelas 2005). Even so, these differences could possibly enable specific genotypes to colonize and survive at the lower depth limit of the vertical distribution range.

Relative gene expression appeared to be more variable within genotypes than the physiological parameters, and no equally clear and consistent effects of shading could be seen in terms of gene expression compared to phenotypic responses. Further, our hypotheses regarding up- or down-regulation of specific genes (Table 1) were accurate only for 
some genotypes. In particular, regulation of genes that were linked to photosynthesis and carbohydrate biosynthesis appeared to be more genotype-specific than light stress-specific (Fig. 4). This could be due to different stress responses between the genotypes or, some genotypes may simply have better resistance to, and recovery from, low irradiance levels. The relative quantification of gene expression can complement studies on phenotypic stress responses. In the current study, we could tentatively link the expression of 2 sucrose metabolism genes, sucrose synthase and sucrose-P-synthase, to changes in plant sucrose concentrations.

Balancing the regulation of growth and carbohydrate reserves in seagrasses is a complicated process including several different pathways. It is surprising that the genotypes up-regulating carbohydrate synthesis related genes (Sphos, STS) the most, were not the same genotypes that up-regulated the photosynthesis-linked genes (Fig. 5); such a trade-off seems paradoxical. Another mismatch appears when examining sucrose concentrations and growth data (Figs. 2 \& 3): exploiting sucrose levels to a greater degree (e.g. Genotype 2) did not increase the specific growth rates of the genotype in question. Neither did this genotype show any signs of using these energy reserves to up-regulate genes related to photosynthesis (Fig. 5). Whether such a reaction is due to differences in energy metabolism or simply due to differences in the capability to rapidly break down and synthesize carbohydrates remains unsolved. That the genotypes consuming most of the storage sucrose during shading (Genotypes 2 and 4) also recovered sucrose levels to control levels and even above during recovery (Figs. $3 \& 4 \mathrm{~b}$ ), suggests a tendency towards more rapid metabolism.

While most of the previous studies have concentrated on differences in stress responses between geographically separated populations (e.g. Bergmann et al. 2010, Franssen et al. 2011, Winters et al. 2011), the current study compared genotypes from the same location. Using the same donor site decreases variation due to local adaptation, but as inter-clone differences represent broad-sense heritability, where individuals may be affected for example by their parents' phenotype (Reusch 2014), such trans-generational effects cannot be completely excluded. The higher significance levels in previous studies (Bergmann et al. 2010, Franssen et al. 2011, Winters et al. 2011) indicates that natural variation in stress responses is lower between single, nearby genotypes than between distant populations consisting of several genotypes. Had we decided to include more genotypes in the study instead of thoroughly studying the responses of 4 genotypes, we most likely would have recorded more local variation. Thus, future studies assessing variation among genotypes should include more genotypes. In addition, as the variation in field experiments tends to be large, we recommend that further experiments aiming to link the carbohydrate metabolism and gene expression should be conducted in a more controlled environment such as aquaria or mesocosms.

To conclude, we addressed genotype-specific variability in stress responses in the field between and within seagrass clones, and found indication of a link between phenotypic changes and gene expression under light-limiting stress. As stress responses and recovery processes can vary substantially between genotypes, this should be considered when planning future stress studies using clonal organisms. The results emphasize the importance of preserving regional genotypic diversity, especially in areas with high clonality.

Acknowledgements. This study was funded by the Åbo Akademi University Endowment (T.S.), GEOMAR Kiel, Svenska kulturfonden (T.S.), Maa- ja vesitekniikan tuki ry., Suomen Luonnonsuojelun Liitto (T.S.), Societas pro Flora et Fauna Fennica (T.S.) and Waldemar von Frenckells stiftelse (T.S.). COST action ES0906: 'Seagrass productivity: from genes to ecosystem management', are acknowledged for funding the stay in Kiel (T.S.). C. Gustafsson, H. Puro, L. Kauppi and E. Savola assisted in the field. K. Beining, A. Beemelmanns, F. Brunner and J. Brakel are thanked for their assistance with the gene expression analysis. G. Banta and M.F. Pedersen kindly provided statistical advice. K. Gagnon is thanked for language revision.

\section{LITERATURE CITED}

Anderson MJ, Gorley RN, Clarke KR (2008) PERMANOVA+ for PRIMER: Guide to software and statistical methods. PRIMER-E, Plymouth

Baden S, Gullström M, Lundén B, Pihl L, Rosenberg R (2003) Vanishing seagrass (Zostera marina, L.) in Swedish coastal waters. Ambio 32:374-377

- Bergmann N, Winters G, Rauch G, Eizaguirre C and others (2010) Population-specificity of heat stress gene induction in northern and southern eelgrass Zostera marina populations under simulated global warming. Mol Ecol 19:2870-2883

Bonsdorff E, Blomqvist EM, Mattila J, Norkko A (1997) Long-term changes and coastal eutrophication. Examples from the Åland Islands and the Archipelago Sea, northern Baltic Sea. Oceanol Acta 20:319-329

Boström C, Baden S, Bockelmann AC, Dromph K and others (2014) Distribution, structure and function of Nordic seagrass ecosystems: implication for coastal management and conservation. Aquatic Conserv: Mar Freshw Ecosyst 24:410-434 
den Hartog C (1970) The sea-grasses of the world. North Holland Publishing, Amsterdam

> Ehlers A, Worm B, Reusch TBH (2008) Importance of genetic diviersity in eelgrass Zostera marina for its resilience to global warming. Mar Ecol Prog Ser 355:1-7

Franssen SU, Gu J, Bergmann N, Winters G and others (2011) Transcriptomic resilience to global warming in the seagrass Zostera marina, a marine foundation species. Proc Natl Acad Sci USA 108:19276-19281

Gustafsson C, Boström C (2013) Influence if neighboring plants on shading stress resistance and recovery of eelgrass, Zostera marina L. PLoS ONE 8:e64064

HELCOM (2009) Eutrophication in the Baltic Sea - an integrated thematic assessment of the effects of nutrient enrichment and eutrophication in the Baltic Sea region. Executive summary. Balt Sea Environ Proc No. 115B

Huber SC, Israel DW (1982) Biochemical basis for partitioning photosynthetically fixed carbon between starch and sucrose in soybean (Glycine max Merr.) leaves. Plant Physiol 69:691-696

Hughes AR, Stachowicz JJ (2004) Genetic diversity enhances the resistance of a seagrass ecosystem to disturbance. Proc Natl Acad Sci USA 101:8998-9002

Hughes AR, Stachowicz JJ (2009) Ecological impacts of genotypic diversity in the clonal seagrass Zostera marina. Ecology 90:1412-1419

Hughes AR, Stachowicz JJ (2011) Seagrass genotypic diversity increases disturbance response via complementarity and dominance. J Ecol 99:445-453

$>$ Hughes AR, Inoye BD, Johnson MTJ, Underwood N, Vellend $M$ (2008) Ecological consequences of genetic diversity. Ecol Lett 11:609-623

> Hughes AR, Stachowicz JJ, Williams SL (2009) Morphological and physiological variation among seagrass (Zostera marina) genotypes. Oecologia 159:725-733

Jump AS, Peñuelas J (2005) Running to stand still: adaptation and the response of plants to rapid climate change. Ecol Lett 8:1010-1020

Nicotra AB, Atkin OK, Bonser SP, Davidson AM and others (2010) Plant phenotypic plasticity in a changing climate. Trends Plant Sci 15:684-692

> Norkko J, Bonsdorff E, Norkko A (2000) Drifting algal mats as an alternative habitat for benthic invertebrates: species specific responses to a transient resource. J Exp Mar Biol Ecol 248:79-104

- Olsen JL, Stam WT, Coyer JA, Reusch TBH and others (2004) North Atlantic phylogeography and large-scale population differentiation of the seagrass Zostera marina L. Mol Ecol 13:1923-1941

Ploner A (2012) Heatplus: Heatmaps with row and/or column covariates and colored clusters. $\mathrm{R}$ package v. 2.8.0

Ransbotyn V, Reusch TBH (2006) Housekeeping gene selection for quantitative real-time PCR assays in the seagrass Zostera marina subjected to heat stress. Limnol Oceanogr Methods 4:367-373

Rasmussen JR, Olesen B, Krause-Jensen D (2012) Effects

Editorial responsibility: Philippe Borsa,

Montpellier, France of filamentous macroalgae mats on growth and survival of eelgrass, Zostera marina, seedlings. Aquat Bot 99: $41-48$

Reusch TBH (2014) Climate change in the oceans: evolutionary versus phenotypically plastic responses of marine animals and plants. Evol Appl 7:104-122

$>$ Reusch TBH, Boström C (2010) Widespread genetic mosaicism in the marine angiosperm Zostera marina is correlated with clonal reproduction. Evol Ecol 25: 899-913

Reusch TBH, Boström C, Stam WT, Olsen J (1999) An ancient eelgrass clone in the Baltic. Mar Ecol Prog Ser 183:301-304

Reusch TBH, Ehlers A, Hämmerli A, Worm B (2005) Ecosystem recovery after climatic extremes enhanced by genotypic diversity. Proc Natl Acad Sci USA 102:2826-2831

> Salo T, Gustafsson C, Boström C (2009) Effects of plant species diversity on primary production and species interactions in brackish water angiosperm communities. Mar Ecol Prog Ser 396:261-272

Sand-Jensen K (1975) Biomass, net production and growth dynamics in an eelgrass (Zostera marina L.) populations in Vellerup Vig, Denmark. Ophelia 14:185-201

Sultan SE (2003) Phenotypic plasticity in plants: a case study in ecological development. Evol Dev 5:25-33

Sultan SE (2004) Promising directions in plant phenotypic plasticity. Perspect Plant Ecol Evol Syst 6:227-233

> Tomas F, Abbott JM, Steinberg C, Balk M, Williams SL, Stachowicz JJ (2011) Plant genotype and nitrogen loading influence seagrass productivity, biogeochemistry, and plant-herbivore interactions. Ecology 92:1807-1817

$>$ Touchette BW, Burkholder JM (2000) Overview of the physiological ecology of carbon metabolism in seagrasses. J Exp Mar Biol Ecol 250:169-205

> Touchette BW, Burkholder JM (2007) Carbon and nitrogen metabolism in the seagrass, Zostera marina L.: Environmental control of enzymes involved in carbon allocation and nitrogen assimilation. J Exp Mar Biol Ecol 350: 216-233

> Villazán B, Pedersen MF, Brun FG, Vergara JJ (2013) Elevated ammonium concentrations and low light form a dangerous synergy for eelgrass Zostera marina. Mar Ecol Prog Ser 493:141-154

Whitehead A, Crawford DL (2006) Neutral and adaptive variation in gene expression. Proc Natl Acad Sci USA 103:5425-5430

Whitham TG, Bailey JK, Schweitzer JA, Shuster SM and others (2006) A framework for community and ecosystem genetics: from genes to ecosystems. Nat Rev Genet 7: 510-523

Winters G, Nelle P, Fricke B, Rauch G, Reusch TBH (2011) Effects of simulated heat wave on photophysiology and gene expression of high- and low-latitude populations of Zostera marina. Mar Ecol Prog Ser 435:83-95

Zar JH (1996) Biostatistical analysis, $3^{\text {rd }}$ edn. Prentice Hall, NJ

Submitted: June 11, 2014; Accepted: October 16, 2014 Proofs received from author(s): December 18, 2014 